\title{
Identification of active thoracic spinal segments responsible for tonic and bursting sympathetic discharge in neonatal rats
}

\author{
Chun-Kuei $\mathrm{Su}^{\mathrm{a}, *}$, Sau-Leong Phoon ${ }^{\mathrm{a}}$, Chen-Tung Yen ${ }^{\mathrm{b}}$ \\ ${ }^{a}$ Institute of Biomedical Sciences, Academia Sinica, Taipei 11529, Taiwan \\ ${ }^{\mathrm{b}}$ Department of Zoology, National Taiwan University, Taipei 10673, Taiwan
}

Accepted 13 December 2002

\begin{abstract}
The isolated thoracic cord of a neonatal rat in vitro generates tonic sympathetic activities in the splanchnic nerves. This tonic sympathetic nerve discharge (SND) has a prominent quasi-periodic oscillation at $\sim 1-2 \mathrm{~Hz}$. Bath application of bicuculline and strychnine, which removes endogenous $\mathrm{GABA}_{\mathrm{A}}$ and glycine receptor activities, transforms the quasi-periodic tonic SND into synchronized bursts (bSND). Picrotoxin, another $\mathrm{GABA}_{\mathrm{A}}$ receptor antagonist, also induces bSND. Serial transections of the thoracic cord (T1-12) were performed to identify the cord segments responsible for these tonic and bursting SNDs. Removal of T1-5 did not affect tonic SND. Nerve-cord preparation with either T6-8 or T10-12 segments could generate a substantial amount of tonic SND that retained comparable oscillating patterns. On the other hand, removal of T1-5 significantly reduced bSND amplitude without affecting its rhythmicity. Either T6-8 or T10-12 segments alone could generate bSND. Mid-point transection of T6-12 at T9 might split bSND rhythmogenesis, leading to the occurrence of bSND that could be attributed to two independent oscillators. Our results demonstrated that three segments within the T6-12 cord were sufficient to generate a rudimentary tonic and bursting SNDs. The thoracic cord segments, however, are dynamically interacting so that a full size bSND could only be produced with the intact thoracic cord.
\end{abstract}

(C) 2002 Elsevier Science B.V. All rights reserved.

Theme: Endocrine and autonomic regulation

Topic: Cardiovascular regulation

Keywords: GABA; Glycine; Synchronization; Gaussian analysis; Propriospinal pathway

\section{Introduction}

Tonic sympathetic nerve discharge (SND) is essential to maintain the basal activities of many visceral organs. Based on the observation that a transection at the cervical spinal cord reduces tonic SND $[2,6,11,22,40]$, it is generally believed that the supraspinal neural structures play a major role in generating this sympathetic tone. However, some studies conversely show that, without supraspinal inputs, the isolated spinal cord still generates a substantial amount of SND [1,17,33,35,39]. The functional roles of such a spinally-generated SND have been shown to be minor under normal physiological conditions [23,27,38]. But, by examining the neural mechanisms responsible for

\footnotetext{
*Corresponding author. Tel.: +886-2-2789-9123; fax: +886-2-27829224.

E-mail address: csu@ibms.sinica.edu.tw (C.-K. Su).
}

the generation of spinal SND, it is very likely that we can gain insights into the basic schemes of sympathetic regulation at the spinal level.

The splanchnic nerves in regulating functions of the abdominal viscera have a significant portion of the tonic SND that seems to be spinally generated. In cats, blockade of higher central inputs by spinal transection did not reduce the splanchnic SND [30,39]. However, in rats, a spinal transection reduced but did not abolish the splanchnic SND [12,41]. Even under in vitro conditions, the spinally ongoing sympathetic activity is present either in the renal nerve or the abdominal sympathetic chain of mice [4], and in the splanchnic nerves of rats [33,34]. Thus, across the species differences in mammals, the supraspinal commands are not essential for the genesis of splanchnic tonic SND.

It is possible that the segmental distribution of the splanchnic sympathetic preganglionic neuron (SPN) con- 
structs the primary frame to generate SND in the splanchnic nerve. Using the retrograde transport of tracers, several studies on different animal species have identified an ipsilateral projection pattern of splanchnic SPNs that mainly extends from the upper to lower thoracic spinal cord $[19,37]$. In hamsters, using the retrograde transport of FluoroGold dye, labeled splanchnic SPNs are found to be located in the spinal segments T3-13 with the greatest number in the T10-12 segments [5]. In rats, splanchnic SPNs are distributed throughout T6-13, with the highest density occurring at T8-9 segments [13]. Similar observations have also been obtained from studying the distribution of SPNs innervating the adrenal gland in rats, wherein the findings indicate that adrenal SPNs are located in T4-13 with a peak distribution at the T8 spinal segment [32]. Although these studies have unraveled the anatomical substrates underlying the central sympathetic outflow to the splanchnic nerves, it remains functionally uncertain what the relative contributions of different spinal segments are to the generation of spontaneous splanchnic SND. The dependence of intersegmental connections to elicit an SND of regular oscillating pattern and the involvement of spinal interneurons in the dynamic recruitment of SPNs from multi-levels of the spinal cord have yet to be explored.

In the isolated thoracic spinal cord of a newborn rat in vitro, power spectral analysis of the splanchnic SND revealed its oscillating patterns, showing a dominant quasiperiodicity $(\sim 1-2 \mathrm{~Hz})$ concomitant with sporadically synchronous bursts $(<0.1 \mathrm{~Hz})$ [34]. The oscillating pattern of this spinally-generated SND is comparable to the dominant rhythm in the discharges of single postganglionic sympathetic neurons innervating the rat tail artery [16]. Using pharmacological tools to block intraspinal $\mathrm{GABA}_{\mathrm{A}}$ and glycine receptor-mediated activities, we found that quasiperiodic SND (qSND) was reduced while bursting SND (bSND) was elicited. The induction of bSND by blocking spinal $\mathrm{GABA}_{\mathrm{A}}$ receptors was also observed in an in vivo study using adult rats [9]. Here, we sought to determine the active segments of the thoracic spinal cord that could generate an SND with regular oscillating pattern. In challenging with $\mathrm{GABA}_{\mathrm{A}}$ and glycine receptors' blockers, we would also determine the minimal spinal segments that contained sufficient neural elements to generate bSND. We anticipated that, by examining the intersegmental dependence of bSND occurrence, our results would unravel the key features of how the splanchnic SPNs that distributed at multi-levels of the spinal cord could synchronize their outflows, so as to transform their output from quasiperiodic to bursting forms.

\section{Materials and methods}

\subsection{General procedures}

Neonatal Sprague-Dawley rats (postnatal days 1-8) were used in this study. Similar to the procedures previously described [33], the thoracic spinal cord (T1-12) was dissected under a microscope and immersed in $10^{\circ} \mathrm{C}$ artificial cerebrospinal fluid (aCSF; in mM: $128 \mathrm{NaCl}, 3$ $\mathrm{KCl}, 1.5 \mathrm{CaCl}_{2}, 1.0 \mathrm{MgSO}_{4}, 24 \mathrm{NaHCO}_{3}, 0.5 \mathrm{NaH}_{2} \mathrm{PO}_{4}$, 30 D-glucose, and 3 ascorbate), equilibrated with $95 \%$ $\mathrm{O}_{2}-5 \% \mathrm{CO}_{2}$. The dorsal parts of the vertebrae encasing the thoracic spinal cord were removed, while the ventral parts of the vertebrae were left intact to preserve the sympathetic efferent pathways. The distal ends of the splanchnic nerves were cut at a level proximal to the celiac ganglion. To record SND, a suction electrode was placed on the proximal end of the whole bundle splanchnic nerves, which contained both the major and minor branches encapsulated by a nerve sheath. During the experiments, the bath temperature was maintained at $24.5 \pm 1{ }^{\circ} \mathrm{C}$.

\subsection{Spinal cord transection}

The preparation was fixed onto the floor of the bath chamber to allow dorsal approaches to the spinal cord. Spinal cord transection was promptly conducted using a microdissecting scissors (Albert Heiss, H-4303). The dorsal roots were used as a landmark for sequential transections. After each transection, the preparation was allowed to rest for $>30 \mathrm{~min}$. The splanchnic SND generated by the spinal segments retained in the bath was compared with the SND obtained prior to the transection to evaluate the relative contribution from different spinal segments to SND genesis.

\subsection{Neural recording and SND signal analysis}

Neural signals recorded from splanchnic SND were amplified, filtered (WPI, DAM50; bandpass: $0.1-1 \mathrm{kHz}$ ), and stored in a PCM-tape recorder (Neuro-Corder, DR890) for off-line analysis. To acquire the envelope of SND ( $\int$ SND), the signals were rectified and integrated using a leaky integrator (discharging time constant: $15 \mathrm{~ms}$ ), thereby revealing the oscillating pattern of SND at a frequency range: 0-67 Hz. Total SND $\left(\int_{0-5} \mathrm{SND}\right)$ was also measured by a time-based integrator (Gould, 13-4615-70) with a resetting time of $5 \mathrm{~s}$. At the end of experiments, the background noise level of SND was determined by integrating the noisy signals after adding $24 \mathrm{mM} \mathrm{Mg}^{2+}$ to the bath solution to block $\mathrm{Ca}^{2+}$-dependent neural activities [10]. True neural signals were then acquired by subtracting the background noise.

A quantitative evaluation of the oscillating pattern of SND was achieved by power spectral analysis of $\int$ SND. We used Pclamp system (Axon Instruments) for data acquisition and Axograph software (version 3.0) for data analysis, wherein $\int$ SND with each episode of the signal registering $40.96 \mathrm{~s}$ was sampled at $0.4 \mathrm{kHz}$. The power spectrum attributed to true neural signals was extracted by subtracting the spectrum of background noise. An average 
of power spectrum for each test in an experiment was acquired from 10-20 episodes. All the power spectra of individual experiments were further normalized to their peak of power at $\sim 1 \mathrm{~Hz}$, when T1-12 spinal segments remained intact.

\subsection{Drugs and applications}

Bicuculline methobromide (Bic, $\mathrm{GABA}_{\mathrm{A}}$ receptor antagonist), picrotoxin (Pic, $\mathrm{GABA}_{\mathrm{A}}$ receptor antagonist), and strychnine hydrochloride (Stry, glycine receptor antagonist) were purchased from Sigma. Concentrated solutions (in mM: $10 \mathrm{Bic}, 4 \mathrm{Pic}$, or 20 Stry) were prepared by dissolving drugs in water and stored at $-20{ }^{\circ} \mathrm{C}$. To elicit a bSND of regular rhythm, a final concentration of $80 \mu \mathrm{M}$ Bic and $10 \mu \mathrm{M}$ Stry in the bath solution was obtained by adding an aliquot of concentrated solutions directly to the bath chamber [34]. A final concentration of $80-160 \mu \mathrm{M}$ Pic was also used to elicit bSND.

\subsection{Gaussian analysis of the Bic- and Stry-induced bSND events}

The variation of the bSND peak amplitude was analyzed by a Gaussian curve fitting algorithm to elucidate if one contiguous section of the spinal cord would generate only one mode of bSND. A plot describing the incidence (i.e. probability of observations) of a bSND that had certain peak amplitude was constructed with the aid of commercially available software, Microcal Origin (version 4.1). First, SND envelope showing recurrent Bic- and Stryinduced bSND signals was low-pass filtered at $1 \mathrm{~Hz}$ to enhance the detection of bSND peak amplitude. We analyzed the consecutive occurrence of bSND events within a 30-min time span. The lowest interburst minimum was used as a zero reference point for the measurement of bSND peak value. Second, the incidence of a bSND that fell into a peak amplitude category was counted. Third, the incidence of a bSND was plotted against its corresponding peak amplitude category. An amplitude version of Gaussian curve fitting algorithm, $y=\underline{A} \cdot \exp \left[-\left(x-x_{\mathrm{c}}\right)^{2} /\left(2 w^{2}\right)\right]$, was then used to fit the data, where $y$ is the probability of observations, $\underline{A}$ is the maximal incidence of the mode, $x_{c}$ is the bSND peak amplitude where the maximal incidence occurs, and $w$ is the width at the half-maximal incidence. The goodness of fit was evaluated by the least chi-square protocol, setting the degrees of freedom (df) as: $\mathrm{df}=k-$ 1 -number of fitted parameters, where $k$ is the number of bSND peak amplitude categories.

\subsection{Data analysis}

To pool the data from individual experiments and minimize variation of electrical recording conditions that may affect the absolute amplitude of neural signals, the integrated SND amplitude under control conditions was considered as $100 \%$ activity. The alteration of integrated SND amplitude after spinal cord transections was then calculated as a percentage change from the control activity. For simple comparisons, paired or unpaired $t$-test was used to verify if neural signals were significantly altered after a treatment. For multiple comparisons, the one-way analysis of variance (ANOVA) was first used to determine the significance, followed by group comparisons using adjusted $t$-tests with $P$-values corrected by Bonferroni methods. The chi-square test was used to evaluate the incidence ratio of certain observations. A $P$-value less than 0.05 was considered significant. All values are presented as means \pm S.E.M.

\section{Results}

\subsection{Oscillating patterns of the spinal SND}

Previous studies using newborn rats 0-2 days old have demonstrated that the thoracic spinal cord in vitro spontaneously generates splanchnic SND [34]. Here, a similar oscillating pattern of the SND was also observed in experiments using rats 1-8 days old. Under control conditions, SND oscillated predominantly in a quasi-periodic form (Figs. 1B and 2B). Power spectral analysis of SND envelope showed the key features of oscillating patterns that contained a major frequency component at $\sim 1-2 \mathrm{~Hz}$.

\subsection{Effects of sequential spinal transections on SND genesis under control conditions}

To assess the segmental contribution of the thoracic spinal cord to SND genesis under control conditions, the spinal cord was sequentially transected and segments of the spinal cord were successively removed. Acute transections at all levels in the thoracic spinal cord often caused a transient SND perturbation that usually subsided within 15 min (Fig. 1). Only the signals acquired in a steady-state are described below.

In rats, the splanchnic SPNs are mainly located in the middle and lower thoracic cord [13,32]. Our first test was to determine whether or not the upper thoracic cord would contribute to the generation of spinal SND. In earlier trials $(n=5)$, the upper thoracic spinal cord (T1-5) was successively transected in a rostrocaudal sequence. Sequential transections made rostral to T5 did not change SND. To minimize the time elapse allowed for regaining a stable SND after the transections, another series of experiments was conducted with the prompt removal of the upper thoracic spinal cord. In this series of experiments $(n=16)$, the T1-5 spinal segments were removed immediately after a transection was made rostral to the T6 dorsal root, leaving T6-12 spinal segments intact. The SND produced by T6-12 spinal cord was comparable to that produced by 

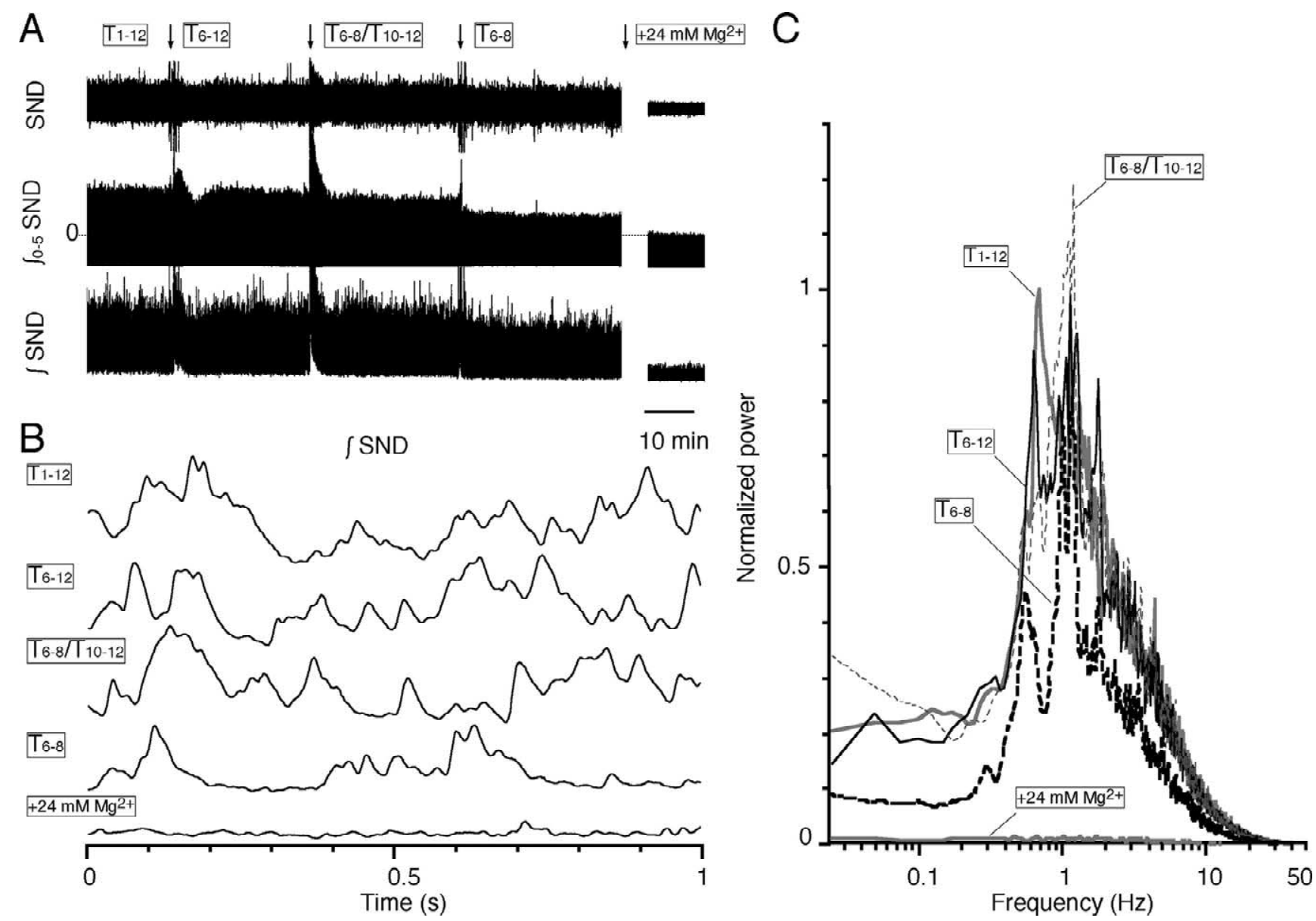

Fig. 1. Effects of sequential transection of the thoracic spinal cord (T1-12) on splanchnic SND: retaining T6-8 spinal segments. (A) Original traces showing the change of SND along the time course of spinal cord transection. The labels on top indicate sections of the spinal cord that remain intact. Arrows from left to right indicate a prompt removal of T1-5 immediately after transectioning at the level rostral to T6 dorsal root, a further transection of T6-12 at the level between T9 and T10 dorsal root, and the removal of T10-12 from T6-8/T10-12 segments (leaving T6-8 intact), respectively. The residual signals after adding $24 \mathrm{mM} \mathrm{Mg}^{2+}$ into the bath reflected the background noise of neural recording (dashed line in the panel of $\int_{0-5} \mathrm{SND}$ ). $\int_{0-5}$ SND: total SND. $\int$ SND: leaky integration of SND. Note that the removal of T1-5 from T1-12 spinal segments did not cause a significant reduction of total SND. The removal of T10-12 from T6-8/T10-12 spinal segments promptly reduced the total SND to $50 \%$ of the control activity. (B) Oscillating patterns of SND as revealed by SND envelope ( $\int$ SND). Ongoing SND with quasi-periodicity persisted throughout the spinal transections until $24 \mathrm{mM}$ $\mathrm{Mg}^{2+}$ was applied. (C) Power spectral analysis of SND envelope. Note the log scales in the horizontal axis for a better display of the power distribution at the lower frequency components $(<0.1 \mathrm{~Hz}$ ). In a plot of normal scales, the power distribution would skew to the left (data not shown). Following spinal transections, the power at all frequency components was not decreased until T10-12 spinal segments were removed from the T6-8/T10-12 spinal segments. The power spectrum of T6-8 spinal segments had a prominent component at $\sim 1 \mathrm{~Hz}$, an activity level comparable to that generated by the T1-12 spinal segments. The power of neural signals was virtually abolished by $24 \mathrm{mM} \mathrm{Mg}{ }^{2+}$.

T1-12 (Figs. 1 and 2). Neither the total SND as estimated by $\int_{0-5}$ SND nor the oscillating pattern as revealed by the power spectrum of SND envelope was altered when T1-5 was removed from the T1-12 spinal segments (Fig. 3).

Subsequently, a further transection of T6-12 spinal cord at T9 (leaving T6-8/T10-12 spinal segments intact) reduced total SND slightly to $67 \pm 6 \%$ of the control activity (the activity generated by T1-12 spinal cord; Fig. $3 \mathrm{~A})$. The transection at $\mathrm{T} 9$ did not significantly alter the oscillating pattern of SND. Fig. 3 shows that the power spectrum of SND envelope generated by T6-8/T10-12 spinal segments remains virtually unchanged at all frequency components, indicating that a separation of T6-12 spinal cord into two independent sections (T6-8/T10-12) did not affect SND genesis.

After the T9 spinal transection, either T6-8 or T10-12 spinal segments were removed to decipher the relative contribution from the retained spinal segments to SND genesis. After removing T10-12 spinal segments, the total SND generated by T6-8 spinal segments retained $50 \pm 7 \%$ $(n=8)$ of the control activity. In contrast, after the removal of T6-8, T10-12 generated only 18 $18 \%(n=8)$ of the control activity, indicating that T6-8 contributes more than T10-12 to the generation of total SND (unpaired $t$-test: $P<0.05)$. To examine the changes of SND oscillating patterns, the power spectra were evaluated quantitatively 


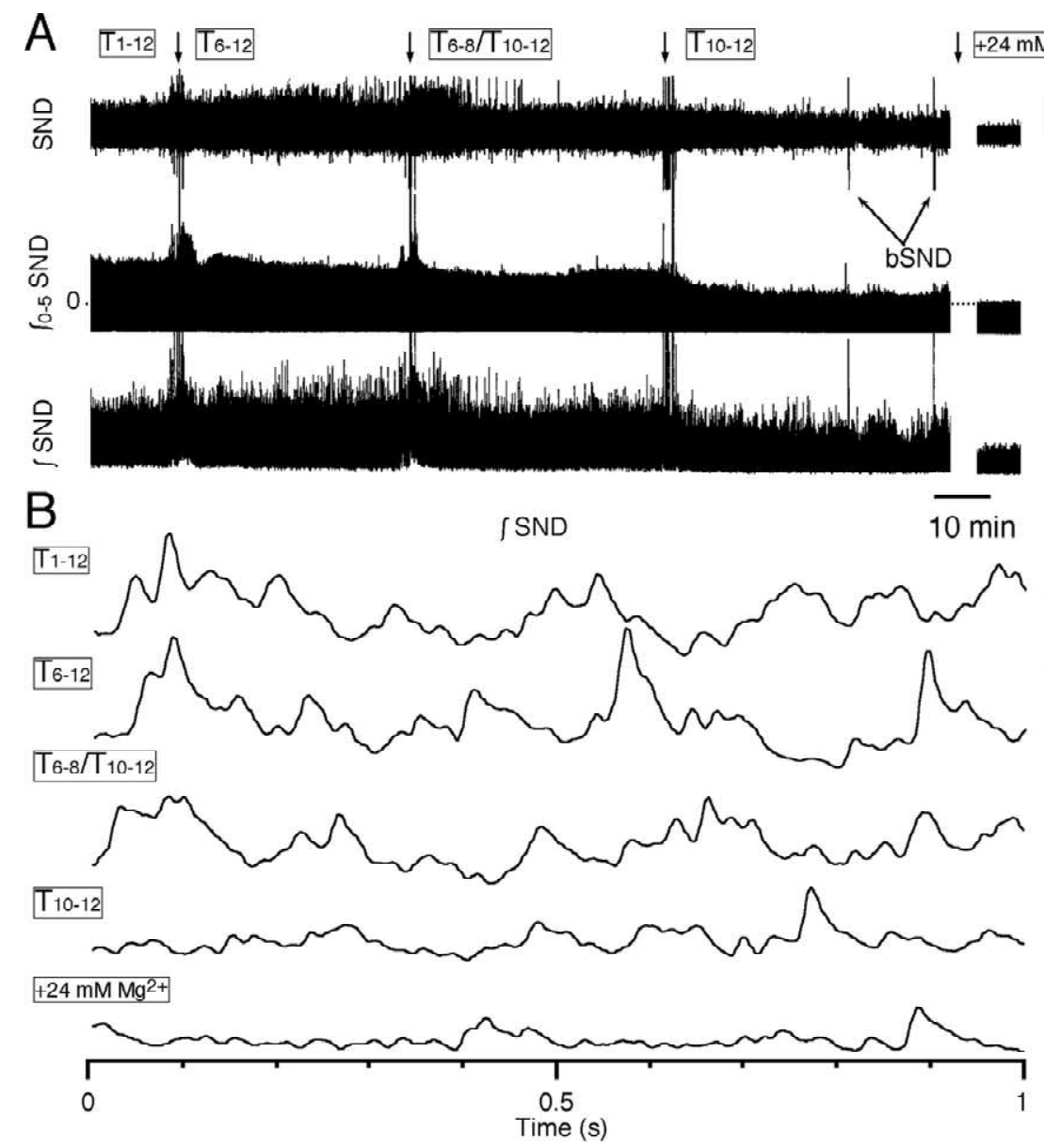

Fig. 2. Effects of sequential transection of the thoracic spinal cord on splanchnic SND: retaining T10-12 spinal segments. A similar paradigm of spinal transections as that shown in Fig. 1 was performed here. (A) T10-12 spinal segments generated an amount of SND that was only $25 \%$ of the control activity. Note also the sporadic occurrence of bursting SND (bSND, arrows). (B) SND envelope showing the diminution of quasi-periodicity when only T10-12 spinal segments remain intact. (C) Power spectral analysis of SND envelope. T10-12 spinal segments generated the SND with an oscillating pattern that was relatively dominant at lower frequency components $(<0.1 \mathrm{~Hz})$, due to an apparent reduction of the power at higher frequency components $(\sim 1 \mathrm{~Hz})$.

by averaging their power at lower $(0.024-0.098 \mathrm{~Hz})$ and higher frequency components $(0.5-2.0 \mathrm{~Hz})$. The data of averaging power from individual experiments were pooled and analyzed as shown in Fig. 3C and D. Removal of either T6-8 or T10-12 spinal segments significantly reduced the power of SND oscillation at $0.5-2 \mathrm{~Hz}$ (Fig. $3 \mathrm{D})$. However, only the removal of T10-12 could significantly reduce the frequency components at $<0.1 \mathrm{~Hz}$ (Fig. 3C).

\subsection{Effects of spinal transections on the bSND genesis induced by Bic and Stry}

To address the segmental contribution of the thoracic spinal cord to bSND genesis, a new series of transection experiments were performed after $80 \mu \mathrm{M}$ Bic and $10 \mu \mathrm{M}$ Stry had been added into the bath solution to elicit bSND. Fig. 4A shows an example where the bSND amplitude decreased after T1-5 was promptly removed from T1-12 spinal cord. Although removing the upper thoracic cord reduced the peak amplitude of bSND (65 $23 \%$ of control activity remained, $P<0.0001, n=7)$, it did not alter bSND rhythm (T1-12 vs. T6-12: $1.05 \pm 0.38$ vs. $1.18 \pm 0.42$ bursts $/ \mathrm{min}, P=0.311, n=7)$.

We further tested if bSND could be elicited by Bic and Stry when only three segments of the lower thoracic spinal cord were kept intact. In one series of experiments $(n=$ 16), Bic and Stry were added into the bath chamber in which only three spinal segments were retained (either T6-8 or T10-12 spinal segments). In the presence of Bic and Stry, qSND was generally reduced while bSND of either regular or irregular rhythm was elicited (Fig. 5). In seven out of the eight trials where the T6-8 spinal segments remained intact, Bic and Stry elicited bSND. In contrast, only in two out of another eight trials, was the bSND with irregular rhythm elicited when T10-12 spinal segments remained intact. When T6-8 remained, the 
A

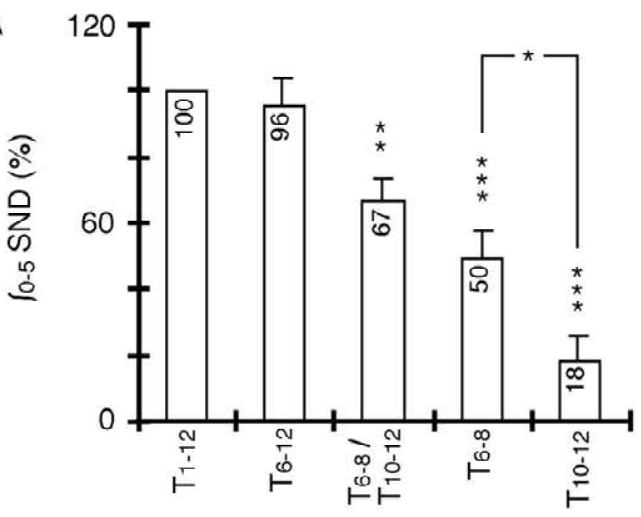

C

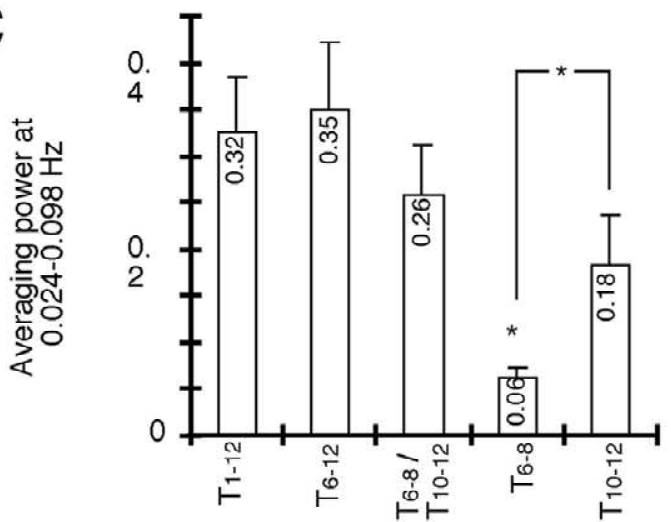

B

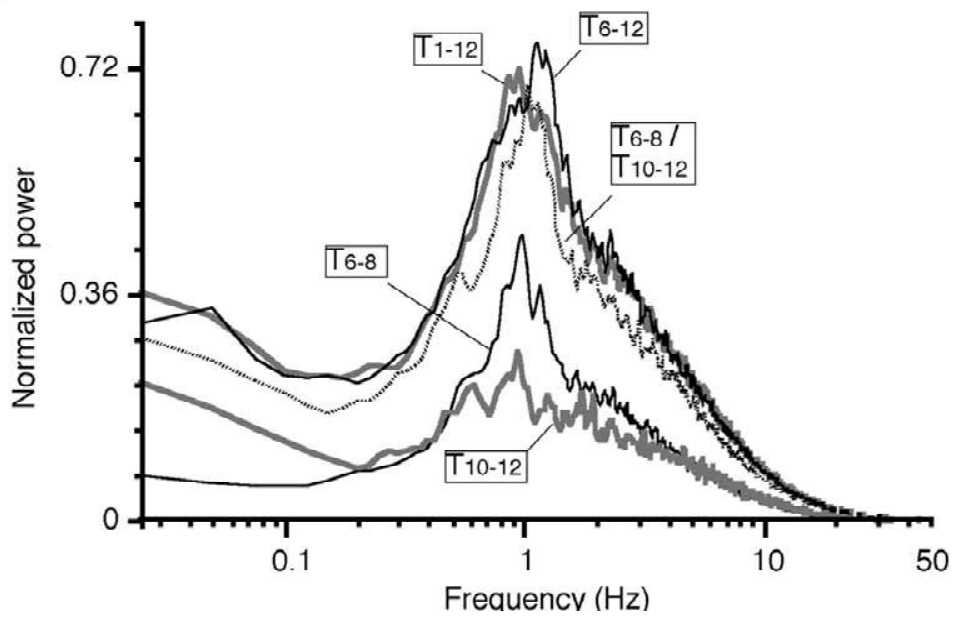

Fig. 3. Relative contribution of the thoracic spinal segments to splanchnic SND. Data shown here were pooled from 16 experiments. Among them, eight experiments retained T6-8 while the other eight experiments retained T10-12 spinal segments. (A) segmental contribution to total SND. T6-12 and T1-12 spinal segments generated similar amount of SND. T6-8/T10-12 spinal segments generated an SND (67 $\pm 6 \%$ ) that was almost equivalent to the summed

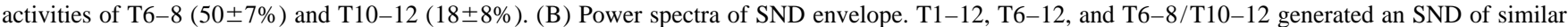
oscillating patterns with comparable powers at all frequency components. In contrast, the power at all frequency components was reduced in T6-8 or T10-12. (C) The lower frequency components of SND generated by T6-8 were significantly smaller than those generated by the other spinal segments. (D) T6-8 or T10-12 generated an SND with significantly reduced power at higher frequency components. Also, note that the spinal segments of T6-12 either with or without a separation at T9 did not significantly decrease their SND power at any frequency components. In A, C, and D, unpaired $t$-test was used to compare the activities generated by T6-8 and T10-12 spinal segments. $* P<0.05$, $* * P<0.01$, and ***P<0.001. ns, not significant.

incidence of eliciting bSND by Bic and Stry was higher ( $\chi^{2}$ test: $\left.P<0.025\right)$.

The possibility that T6-8 and T10-12 spinal segments in the same preparation could independently generate bSND was also tested by promptly transecting T6-12 spinal cord at T9 $(n=10)$. In seven out of ten trials, a transection at $\mathrm{T} 9$ reduced the bSND peak amplitude to $68 \pm 6 \%$ of that generated by T6-12 $(P<0.005)$, but the transection did not apparently affect bSND rhythmicity (Fig. 5). In the other three trials, distinct bSND events with irregular amplitude and rhythmicity were elicited by the transection (Fig. 6). In these three trials, a variation analysis of the bSND peak amplitude was therefore used to demonstrate that the transection of T6-12 spinal segments at T9 caused a split of bSND rhythmogenesis.

\subsection{Variation analysis of the bSND peak amplitude}

The Bic- and Stry-induced bSND often showed substantial variations in amplitude (Fig. 4A). We anticipated that, if bSND was generated by the spinal neurons which synchronized outputs through their intersegmental connections, a contiguous section of the spinal cord would generate a peak amplitude of bSND that varied within a 'normal' range. Gaussian curve fitting algorithm was therefore used to fit the incidence plot of bSND peak amplitude. Among all the tests, the incidence of bSND generated by one contiguous section of the thoracic spinal cord (either T1-12 or T6-12) complied with an unimodal Gaussian curve (Fig. 4). On the other hand, in three out of ten trials where a transection of T6-12 spinal segments at 


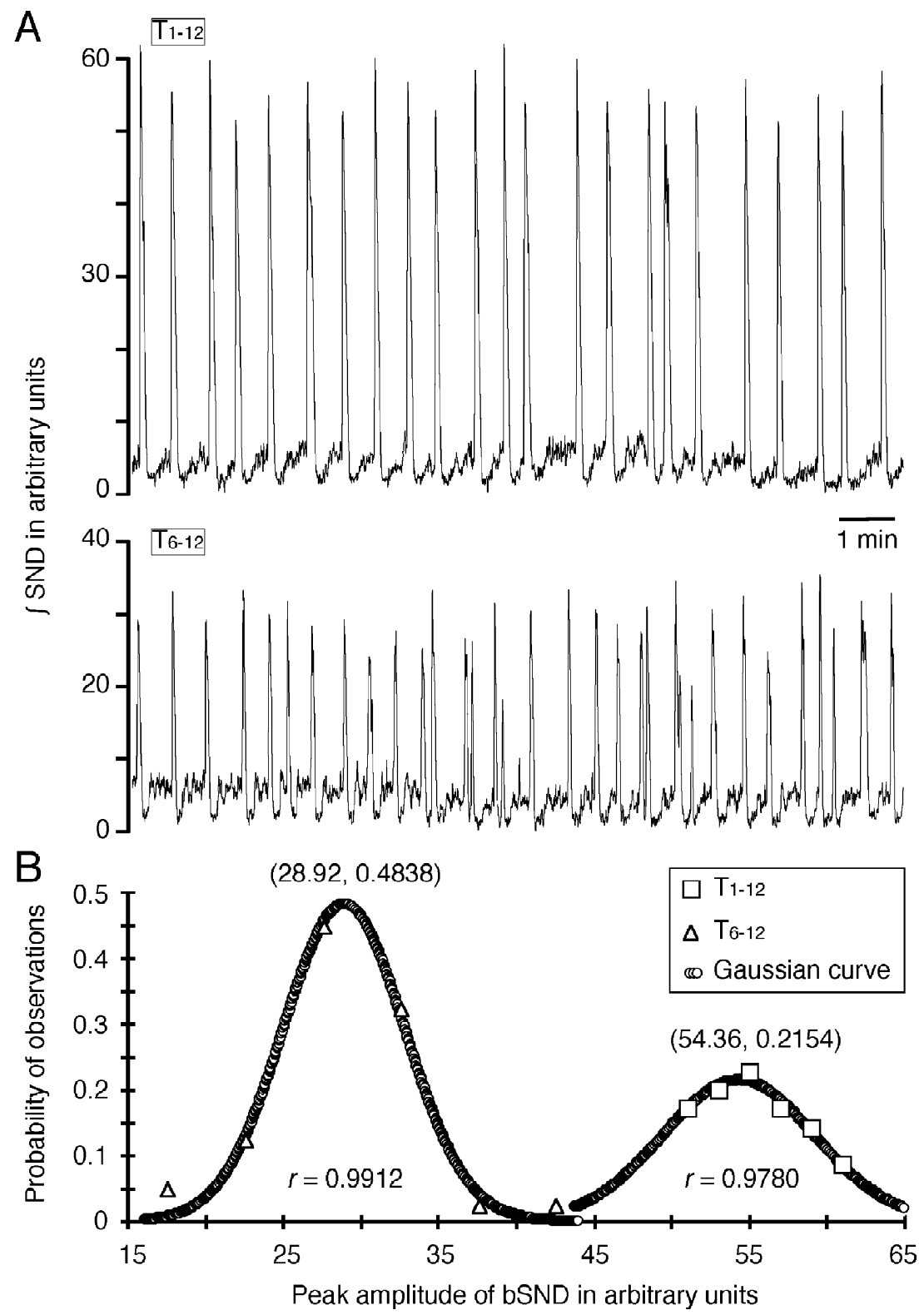

Fig. 4. The alteration of Bic- and Stry-induced bSND after removal of T1-5 from T1-12 spinal segments. The bSND was induced by $80 \mu M$ Bic and 10 $\mu \mathrm{M}$ Stry. (A) bSND generated by T1-12 (top panel) and T6-12 spinal segments (lower panel). Removal of T1-5 from T1-12 spinal segments reduced the bSND peak amplitude by $\sim 50 \%$. The rhythmicity of bSND was not affected. (B) The incidence plot of bSND peak amplitude. The lines formed by the open circles represent the Gaussian curves fitted for the data of T1-12 or T6-12. The incidence plots were based on 35 or 40 bSND events with their peak amplitude being ranked into six categories (in arbitrary units: range from 51 to 61 or 17.5 to 42.5 with an incremental step of 2 or 5 for the events of T1-12 or T6-12 spinal segments). $r$, the correlation coefficient of the data fitting with an unimodal Gaussian function: $y=A \cdot \exp \left[-\left(x-x_{\mathrm{c}}\right)^{2} /\left(2 w^{2}\right)\right]$. The values in parentheses on top of the mode are $x_{\mathrm{c}}$ and $\underline{A}$. The $w$ for T1-12 and T6-12 spinal segments were 4.8856 and 3.9164, respectively. The goodness of fit was evaluated by the least chi-square (T1-12: 0.00018, T6-12: 0.00094), where the degrees of freedom (df) for both were calculated as: number of categories $(6)-$ number of parameters fitted $(3)-1=2$. Thus, both for T1 -12 and T6-12, the $P$-value in rejecting the hypothesis that the data fitted well with a Gaussian distribution was less than 0.005 .

T9 succeeded in eliciting a bSND of irregular patterns, the incidence plots fit well with a bi- or trimodal Gaussian curve. Fig. 6 shows an example where the incidence of bSND peak amplitude (generated by T6-8/T10-12 spinal segments) was well-described by a trimodal Gaussian function (Fig. 6C, $r=0.99985$ ). Notably, the third mode with the largest bSND peak amplitude at 79.96 had an incidence of 0.0705 , a value approximating a cross product of the incidence from the other two modes $(0.4242 \times$ $0.1795=0.0761$ ). This suggests that the occurrence of the third mode is dependent on the incidence of the other two modes. After T6-8 spinal segments were removed, the 

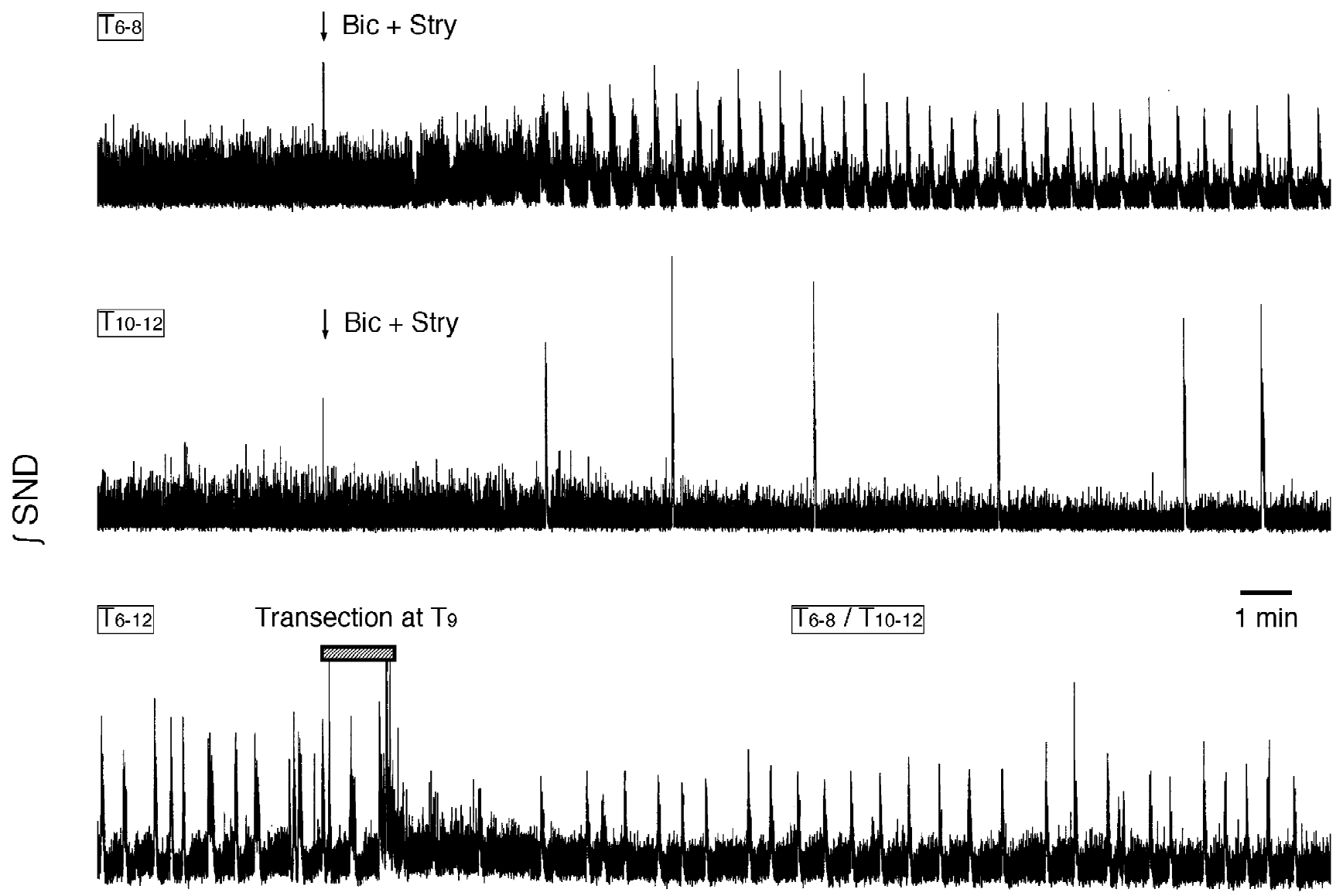

Fig. 5. Bic and Stry effects on the SND generated by T6-8 or T10-12 spinal segments. Each panel shows the responses of an individual experiment. (Upper panel) A bSND of regular rhythm was induced by application of Bic and Stry to T6-8 spinal segments. (Middle panel) Bic and Stry induced a sporadic bSND of higher amplitude in T10-12 spinal segments. (Lower panel) A transection of T6-12 spinal segments at a level between T9 and T10 dorsal roots reduced bSND amplitude. Data only show that Bic and Stry have been added into the bath to elicit bSND. In this example, the origination of bSND from either T6-8 or T10-12 spinal segments could not be easily distinguished by examining its peak amplitude.

data acquired from T10-12 spinal segments were distributed only in the first mode, confirming that the first mode of bSND events was generated by the T10-12 spinal segments. For the other two trials (data not shown), the bSND events generated by T6-8/T10-12 spinal segments had a relatively similar peak amplitude. A considerable overlapping distribution of bSND peak amplitude was therefore observed in their incidence plots. In these two trials, the bSND events generated by T6-8/T10-12 spinal segments were best fitted with bimodal Gaussian curves $(r=0.98302$ or 0.99115$)$.

\subsection{The bSND induced by Pic}

The quaternary salts of Bic have been shown to alter neuronal excitability via non-GABA $\mathrm{A}_{\mathrm{A}}$ receptor-mediated effects [28,31]. Although the main task here was not aimed at determining if Bic-induced bSND was exclusively due to a blockade of endogenous $\mathrm{GABA}_{\mathrm{A}}$ receptor-mediated activities, it was imperative to clarify if bSND could be elicited by another more specific $\mathrm{GABA}_{\mathrm{A}}$ receptor antagonist. In a dissociated cell preparation, unlike $100 \mu \mathrm{M}$
Bic-methiodide, $100 \mu \mathrm{M}$ Pic did not affect $\mathrm{Ca}^{2+}$-activated $\mathrm{K}^{+}$current [15]. Thus, we examined if Pic at a dose of $\sim 100 \mu \mathrm{M}$ could induce bSND. Fig. 7 shows an example where Pic at a dose as low as $80 \mu \mathrm{M}$ induces bSND. However, the induction of bSND with such a low dose of Pic was inconsistent. Only three out of 12 experiments (25\%) using $80 \mu \mathrm{M}$ Pic were successful in eliciting bSND. When the doses of Pic were cumulatively raised to 100 , 120 , and $160 \mu \mathrm{M}$, the ratios of successful trials in eliciting bSND were increased $(30,58$, and $83 \%$, respectively). Since Pic at a dose of $100 \mu \mathrm{M}$ could mimic the effects of $80 \mu \mathrm{M}$ Bic in inducing bSND, the occurrence of bSND in this study was, at least partly, attributable to a blockade of endogenous $\mathrm{GABA}_{\mathrm{A}}$ receptor-mediated activities.

\section{Discussion}

\subsection{Active spinal segments responsible for the generation of splanchnic SND}

Under in vitro conditions, we found that T6-12 spinal 


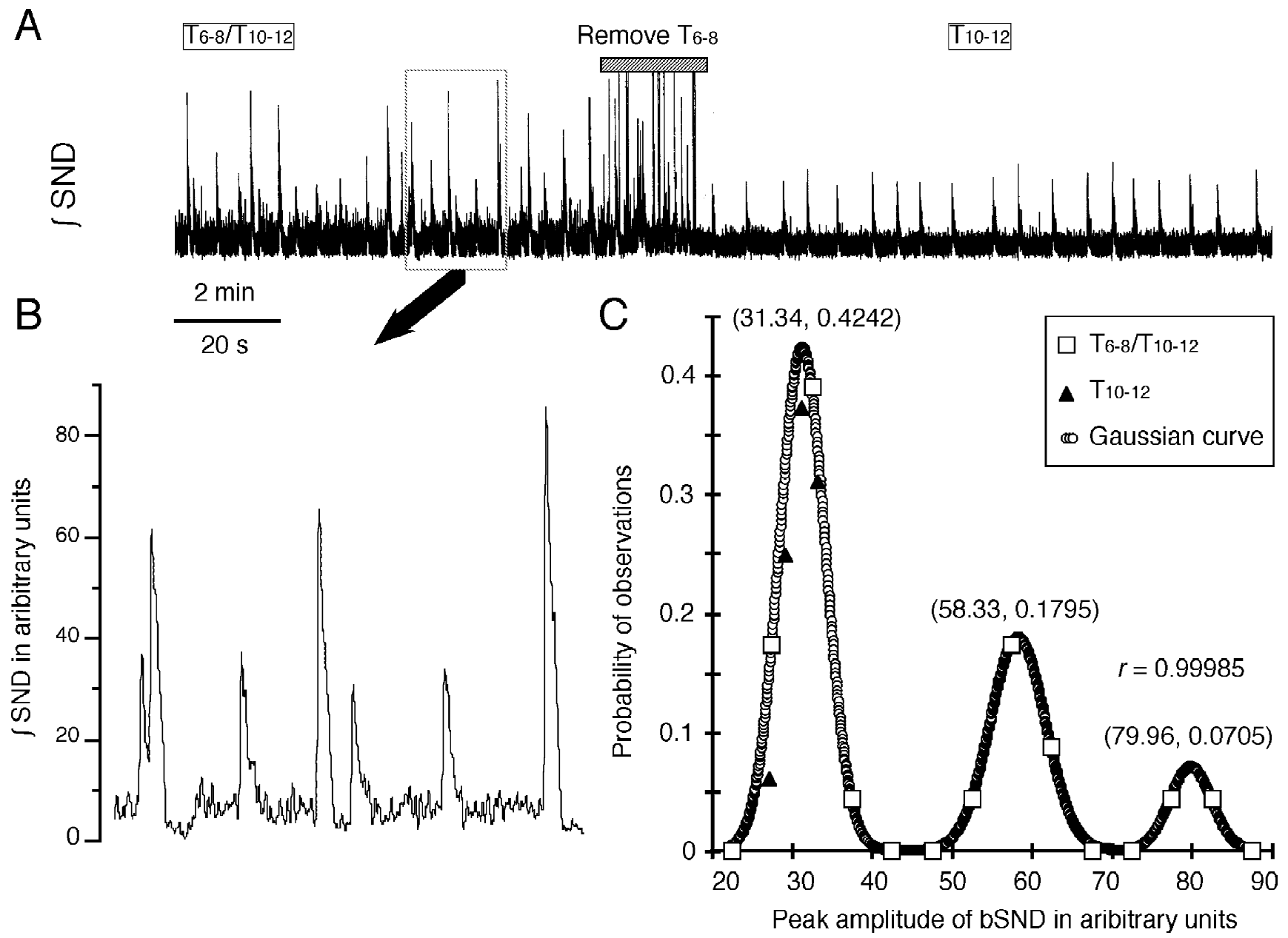

Fig. 6. Distinct Bic- and Stry-induced bSND events generated by T6-8/T10-12 spinal segments. (A) Two types of bSND generated by T6-8/T10-12 spinal segments. T6-8/T10-12 spinal segments generated bSNDs that varied greatly in amplitude. After T6-8 spinal segments were removed, only the bSND events of smaller amplitude persisted in a more regular rhythm. (B) The faster trace showing the bSND of smaller amplitude appeared concomitantly with that of larger amplitude. (C) The incidence plot of bSND peak amplitude. The incidence plots were based on 23 or 16 bSND events generated by either T6-8/T10-12 or T10-12 spinal segments. For bSND events of T6-8/T10-12 or T10-12 spinal segments, their peak amplitude in arbitrary units was ranking from 22.5 to 87.5 or from 27 to 33 with an incremental step of 5 or 2 , giving 14 or four categories. A trimodal amplitude version of Gaussian function (for $i=1$ to $3, y=\sum A_{i} \cdot \exp \left[-\left(x-x_{c i}\right)^{2} /\left(2 w_{i}^{2}\right)\right]$ ) was used to fit the data of T6-8/T10-12 spinal segments (the line formed by open circles). The fit yielded a correlation coefficient, $r=0.99985$. The values in parentheses on top of each mode represent $x_{c \mathrm{i}}$ and $\underline{A}_{i}$. From left to right, the $w_{i}$ were $2.8781,3.4395$, and 2.5896 , respectively. The goodness of fit was indicated by a least chi-square equal to $0.0000097(\mathrm{df}=14-9-1=4)$. The $P$-value in rejecting the hypothesis that the data fitted well with the trimodal Gaussian distribution was far less than 0.005 .

segments generated an SND almost equivalent to the SND generated by $\mathrm{T} 1-12$. This finding suggests that, under control conditions, T1-5 spinal segments are not tonically active in supplying inputs to the SPNs. However, in the presence of Bic and Stry, the contribution from T1-5 to bSND genesis was apparent, because the peak amplitude of bSND was significantly reduced after removing T1-5 from T1-12. Thus, it seems that the SPN-driving neurons in T1-5 spinal segments are normally under endogenous inhibition mediated by $\mathrm{GABA}_{\mathrm{A}}$ and glycine receptors. When these endogenous activities are reduced, the upper thoracic segments can actively participate in bSND genesis.

When would the intraspinal GABA and glycine neurotransmitter activities be reduced under physiological con- ditions? At the spinal level, glycine mediates the baroreceptor inhibition of SND [20], while GABA working via $\mathrm{GABA}_{\mathrm{A}}$ receptors depresses somatosympathetic reflex [9]. In spinal rats, the loss of tonic firing in the renal nerve after dorsal rhizotomy could be counteracted by a mechanism intrinsic to the spinal cord [36]. Thus, it is likely that the loss of the glycine-mediated baroreflex inhibition of SND or GABA-mediated inhibition of somatosympathetic reflex may occur when the baroreceptor or somatic inputs are depressed (e.g. acute hypotension). A sudden fall of the intraspinal glycine or $\mathrm{GABA}_{\mathrm{A}}$ receptor-mediated activities might in turn prompt another neural mechanism to recruit the available SND-excitatory neurons located in those quiescent spinal segments. This can in part account for the partial compensation of the loss of tonic firing in renal 


\section{Control}
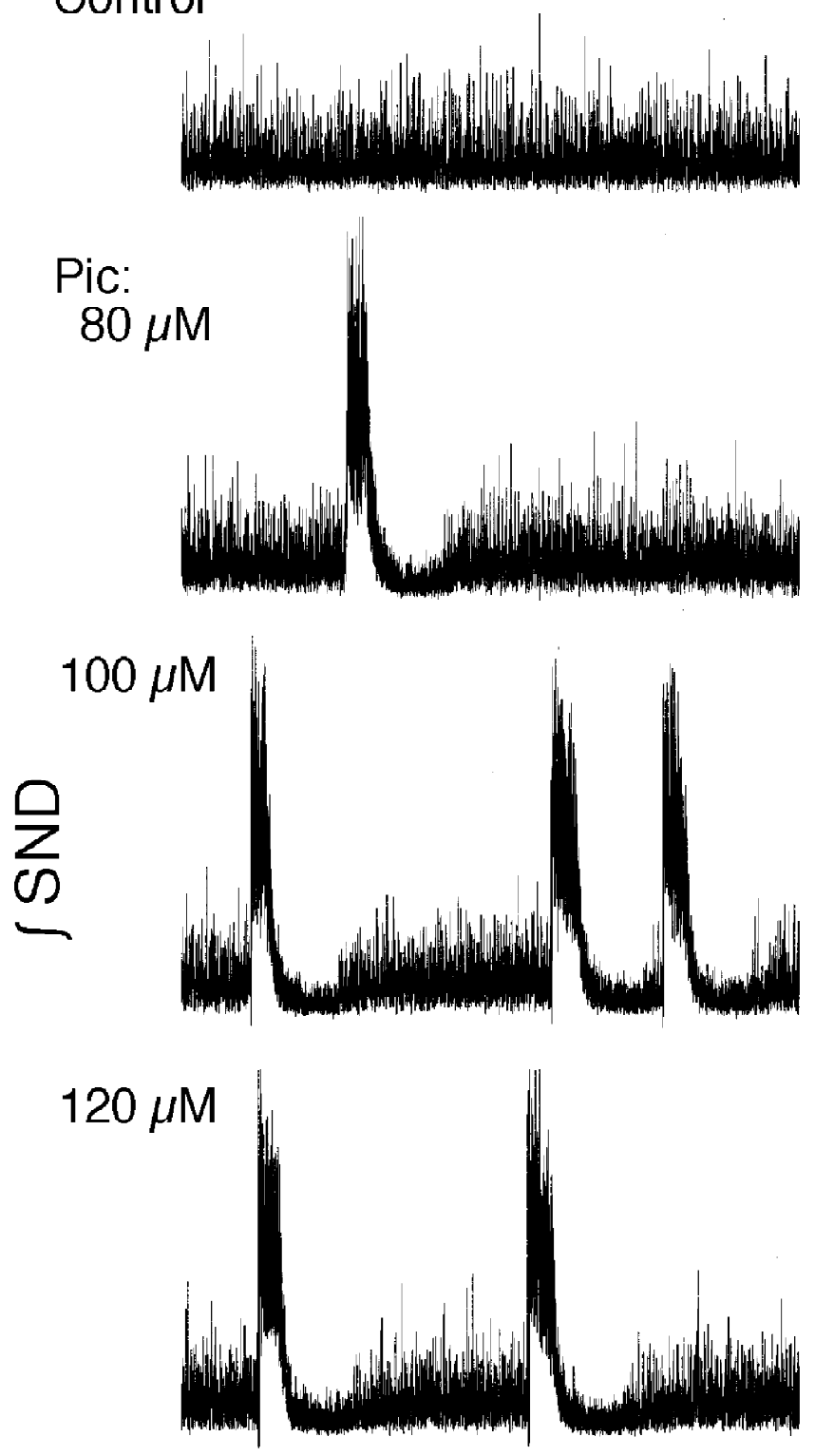

$+24 \mathrm{mM} \mathrm{Mg}^{2+}$

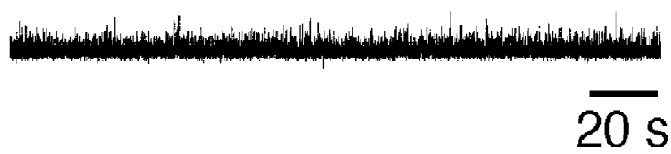

Fig. 7. Pic induces bSND. Original traces showing the induction of bSND by $80-120 \mu \mathrm{M}$ Pic. In the presence of $24 \mathrm{mM} \mathrm{Mg}^{2+}$, both tonic and bursting components of SND are abolished.

SND after dorsal rhizotomy [36]. It also provides useful clues to understand the display of cardiovascular Mayer waves in the initial compensatory phase of hemorrhage $[21,29]$ or the enhancement of low frequency SND oscillation in response to a diminution of baroreceptor inputs during aortic constriction in spinal cats [24]. Notably, the active spinal segments that maintain tonic activities of the cardiac and vertebral sympathetic nerves are not as widely distributed as the distribution of all SPNs available for controlling the targets supplied by these two nerves [18]. Consistent with this finding is that, although the distribution of splanchnic SPNs in rats may extend from the upper to the lower thoracic cord [13,32], the upper thoracic cord (T1-5 spinal segments) is primarily quiescent under control conditions. Only when the intraspinal $\mathrm{GABA}_{\mathrm{A}}$ and glycine receptors-mediated activities are blocked, will the upper thoracic cord become active for bSND genesis. Our results suggest that the upper thoracic cord may serve as a dynamic repertoire to enhance the synchronous output of splanchnic SND.

\subsection{Differential characteristics of discharging patterns in adjacent spinal segments}

The data, obtained after a transection at T9 to separate T6-12 into T6-8/T10-12 spinal segments, indicate that these two sections of the spinal cord contribute differently to the generation of splanchnic SND. Compared to those activities generated by T10-12, T6-8 spinal segments generated more total SND but less power in the lower frequency components (Fig. 3). Moreover, a blockade of $\mathrm{GABA}_{\mathrm{A}}$ and glycine receptor-mediated activities in T6-8 spinal cord resulted in a higher incidence of eliciting bSND. These observations imply that T6-8 and T10-12 spinal cord may contain different proportions of functionally distinct subsets of splanchnic SPNs that play different roles in regulating either visceral vasoconstriction or the motility of pelvic organs $[14,25]$. Since the segmental control of specific visceral targets requires only adjacent spinal segments [26], the difference in discharging patterns between $\mathrm{T} 6-8$ and $\mathrm{T} 10-12$ may reflect the functional differentiation of their controls over target organs.

\subsection{Three or less intersegmental connections are sufficient for SND genesis}

Under control conditions, a transection of T6-12 spinal cord at $\mathrm{T} 9$ to separate the cord into $\mathrm{T} 6-8 / \mathrm{T} 10-12$ segments does not affect the oscillating patterns of SND, even though the transection significantly reduces total SND (Fig. 3). This observation implies that the generation of SND in quasi-periodic forms does not entirely depend on the intersegmental connections across the T9 spinal segment. Either the neural mechanisms in the T9 segment or those mechanisms requiring intersegmental connections across T9 contribute only to total SND, but not to the SND oscillating patterns. Although we could not determine how much neural component was severed when the cord was transected at $\mathrm{T} 9$, the persistence in the quasi-periodicity of SND as revealed by a similar power spectrum between T6-12 and T6-8/T10-12 suggests that the intersegmental 
connection of more than three segments is not essential for generating an SND with normal oscillating patterns.

It is intriguing that, in the presence of Bic and Stry, a contiguous section of the spinal cord generates an unimodal bSND. Such unimodal bSND events could be dissociated into multimodes after a contiguous section of the cord was further separated into individual segments. The unimodal distribution of bSND events indicates that the neural circuits located in every individual spinal segments are normally coupled with each other through intersegmental connections. Upon separation, the individual spinal segments spontaneously generate bSNDs of their own, thereby giving rise to multimodal bSND events (Fig. 6). All these activities in bursting forms could have been well-coordinated by the propriospinal circuitry, which requires as few as three spinal segments. For a section of the cord longer than three segments, the cross-talk between neighboring segments could then integrate the sympathetic outflow, allowing an unimodal characteristics of synchronous bursts.

\section{Conclusions}

The interconnections of subsets of neurons within and between spinal segments construct the basic frame to elicit an SND of oscillating patterns. In our previous study, we have reported that intraspinal glutamate, GABA, and glycine activities are involved in the generation of spontaneous splanchnic SND [34]. Here, we further clarify that under control conditions, T6-12 spinal segments are the most active segments for the generation of splanchnic SND. Since the neural circuits in three segments of the lower thoracic cord are sufficient to generate a normal oscillating pattern of SND, which can transform into bursts under certain conditions, we propose that three or less spinal segments formulate the basic operational units for SND genesis. Through intersegmental connections, the circuitry within individual units could be integrated and the available SPNs can be dynamically recruited. Consistent with the idea that segmental control of specific visceral targets requires only adjacent spinal segments $[18,26]$, the present evidence strongly supports the concept that such a controlling system could either be dynamically integrated or selectively commanded $[3,7,8,11,35]$.

\section{Acknowledgements}

We are grateful to Shu-Chun Peng and Yi-Wen Cheng for technical assistance. This research was supported by grants from the Institute of Biomedical Sciences, Academia Sinica, and National Science Council of the Republic of China (NSC 90-2320-B-001-043).

\section{References}

[1] R.S. Alexander, Tonic and reflex functions of medullary sympathetic cardiovascular centers, J. Neurophysiol. 9 (1946) 205-217.

[2] A.M. Allen, J.M. Adams, P.G. Guyenet, Role of the spinal cord in generating the 2- to 6-Hz rhythm in rat sympathetic outflow, Am. J. Physiol. 33 (1993) R938-R945.

[3] H.-S. Chang, K. Staras, J.E. Smith, M.P. Gilbey, Sympathetic neuronal oscillators are capable of dynamic synchronization, J. Neurosci. 19 (1999) 3183-3197.

[4] B.A. Chizh, P.M. Headley, J.F.R. Paton, Coupling of sympathetic and somatic motor outflows from the spinal cord in a perfused preparation of adult mouse in vitro, J. Physiol. (Lond.) 508 (1998) 907-918.

[5] N.S. Dehal, G.A. Dekaban, A.V. Krassioukov, F.J. Picard, L.C. Weaver, Identification of renal sympathetic preganglionic neurons in hamsters using transsynaptic transport of herpes simplex type I virus, Neuroscience 56 (1993) 227-240.

[6] G.L. Gebber, S.M. Barman, M. Zviman, Sympathetic activity remains synchronized in the presence of a glutamate antagonist, Am. J. Physiol. 256 (1989) R722-R732.

[7] G.L. Gebber, S. Zhong, S.M. Barman, Synchronization of cardiacrelated discharges of sympathetic nerves with inputs from widely separated spinal segments, Am. J. Physiol. 268 (1995) R1472R1483.

[8] M.P. Gilbey, Multiple oscillators, dynamic synchronization and sympathetic control, Clin. Exp. Pharmacol. Physiol. 28 (2001) $130-137$.

[9] A.K. Goodchild, B.T.M. Van Deurzen, Q.-J. Sun, J. Chalmers, P.M. Pilowsky, Spinal GABA A receptors do not mediate the sympathetic baroreceptor reflex in the rat, Am. J. Physiol. 279 (2000) R320R331.

[10] P. Grafe, C.J. Mayer, J.D. Wood, Synaptic modulation of calciumdependent potassium conductance in myenteric neurons in the guinea-pig, J. Physiol. (Lond.) 305 (1980) 235-248.

[11] K. Hayes, L.C. Weaver, Selective control of sympathetic pathways to the kidney, spleen and intestine by the ventrolateral medulla in rats, J. Physiol. (Lond.) 428 (1990) 371-385.

[12] K. Hayes, C.P. Yardley, L.C. Weaver, Evidence for descending tonic inhibition specifically affecting sympathetic pathways to the kidney in rats, J. Physiol. (Lond.) 434 (1991) 295-306.

[13] Y. Hong, L.C. Weaver, Distribution of immunoreactivity for enkephalin, substance $\mathrm{P}$ and vasoactive intestinal peptide in fibers surrounding splanchnic sympathetic preganglionic neurons in rats, Neuroscience 57 (1993) 1121-1133.

[14] W. Jänig, E.M. McLachlan, Organization of lumbar spinal outflow to distal colon and pelvic organs, Physiol. Rev. 67 (1987) 1332-1404.

[15] S. Johansson, M. Druzin, D. Haage, M.-D. Wang, The functional role of a bicuculline-sensitive $\mathrm{Ca}^{2+}$-activated $\mathrm{K}^{+}$current in rat medial preoptic neurons, J. Physiol. (Lond.) 532 (3) (2001) 625635.

[16] C.D. Johnson, M.P. Gilbey, On the dominant rhythm in the discharges of single postganglionic sympathetic neurones innervating the rat tail artery, J. Physiol. (Lond.) 497 (1) (1996) 241-259.

[17] M.J. Kenney, J.G. Pickar, M.L. Weiss, C.S. Saindon, R.J. Fels, Effects of midbrain and spinal cord transections on sympathetic nerve responses to heating, Am. J. Physiol. 278 (2000) R1329R1338.

[18] B. Kocsis, K. Gyimesi-Pelczer, Spinal segments communicating resting sympathetic activity to postganglionic nerves of the stellate ganglion, Am. J. Physiol. 275 (1998) R400-R409.

[19] D.C. Kuo, D.S. Yamasaki, G.M. Krauthamer, Segmental organization of sympathetic preganglionic neurons of the splanchnic nerve as revealed by retrograde transport of horseradish peroxidase, Neurosci. Lett. 17 (1980) 11-16.

[20] D.I. Lewis, J.H. Coote, Mediation of baroreceptor inhibition of 
sympathetic nerve activity via both a brainstem and spinal site in rats, J. Physiol. (Lond.) 481 (1994) 197-205.

[21] S.C. Malpas, D.E. Burgess, Renal SNA as the primary mediator of slow oscillation in blood pressure during hemorrhage, Am. J. Physiol. 279 (2000) H1299-H1306.

[22] R.L. Meckler, L.C. Weaver, Splenic, renal, and cardiac nerves have unequal dependence upon supraspinal inputs, Brain Res. 338 (1985) $123-135$.

[23] C.O. Miller, D.G. Johns, L.P. Schramm, Spinal interneurons play a minor role in generating ongoing renal sympathetic nerve activity in spinally intact rats, Brain Res. 918 (2001) 101-106.

[24] N. Montano, C. Cogliati, V.J. da Silva, T. Gnecchi-Ruscone, M. Massimini, A. Porta, A. Malliani, Effects of spinal section and positive-feedback excitatory reflex on sympathetic and heart rate variability, Hypertension 36 (2000) 1029-1034.

[25] S.F. Morrison, D.J. Reis, Responses of sympathetic preganglionic neurons to rostral ventrolateral medullary stimulation, Am. J. Physiol. 261 (1991) R1247-R1256.

[26] A. Nja, D. Purves, Specific innervation of guinea-pig superior cervical ganglion cells by preganglionic fibers arising from different levels of the spinal cord, J. Physiol. (Lond.) 264 (1977) 565-583.

[27] J.W. Osborn, R.H. Livingstone, L.P. Schramm, Elevated renal nerve activity after spinal transection: effects on renal function, Am. J. Physiol. 253 (1987) R619-R625.

[28] J.-F. Pflieger, F. Clarac, L. Vinay, Picrotoxin and bicuculline have different effects on lumbar spinal networks and motoneurons in the neonatal rat, Brain Res. 935 (2002) 81-86.

[29] G. Preiss, C. Polosa, Patterns of sympathetic neuron activity associated with Mayer waves, Am. J. Physiol. 226 (1974) 724-730.

[30] L. Qu, R. Sherebrin, L.C. Weaver, Blockade of spinal pathways decreases pre- and postganglionic discharge differentially, Am. J. Physiol. 255 (1988) R946-R951.

[31] V. Seutin, S.W. Johnson, Recent advances in the pharmacology of quaternary salts of bicuculline, Trends Pharmacol. Sci. 20 (1999) 268-270.
[32] A.M. Strack, W.B. Sawyer, K.B. Platt, A.D. Loewy, CNS cell groups regulating the sympathetic outflow to adrenal gland as revealed by transneuronal cell body labeling with pseudorabies virus, Brain Res. 491 (1989) 274-296.

[33] C.-K. Su, Rhythmic sympathetic nerve discharges in an in vitro neonatal rat brainstem-spinal cord preparation, J. Appl. Physiol. 87 (1999) 1066-1075.

[34] C.-K. Su, Intraspinal amino acid neurotransmitter activities are involved in the generation of rhythmic sympathetic nerve discharge in newborn rat spinal cord, Brain Res. 904 (2001) 112-125.

[35] R.F. Taylor, L.P. Schramm, Differential effects of spinal transection on sympathetic nerve activities in rats, Am. J. Physiol. 253 (1987) R611-R618.

[36] R.B. Taylor, L.C. Weaver, Dorsal root influences on tonic firing of renal and mesenteric sympathetic nerve activities in rats, Am. J. Physiol. 264 (1993) R1193-R1199.

[37] Y. Torigoe, R.D. Cernucan, J.A.S. Nishimoto, R.H.I. Blanks, Sympathetic preganglionic efferent and afferent neurons mediated by the greater splanchnic nerve in rabbit, Exp. Neurol. 87 (1985) 334-348.

[38] K.A. Trostel, J.W. Osborn, Does the spinal cord generate functionally significant sympathetic activity in the awake rat?, Am. J. Physiol. 266 (1994) R1102-R1110.

[39] L.C. Weaver, R.D. Stein, Effects of spinal cord transection on sympathetic discharge in decerebrate-unanesthetized cats, Am. J. Physiol. 257 (1989) R1506-R1511.

[40] S. Zhong, M.J. Kenney, G.L. Gebber, Higher power, lower frequency components of cardiac, renal, splenic and vertebral sympathetic nerve activities are uniformly reduced by spinal cord transection, Brain Res. 556 (1991) 130-134.

[41] S. Zhong, Z.S. Huang, G.L. Gebber, S.M. Barman, Role of the brain stem in generating the 2- to $6-\mathrm{Hz}$ oscillation in sympathetic nerve discharge, Am. J. Physiol. 265 (1993) R1026-R1035. 